\title{
Treatment-responsive, reversible, autoimmune encephalitis in a child
}

\author{
J Wanigasinghe ${ }^{1}$, T Chang $^{2}$, A Vincent ${ }^{3}$ \\ Ceylon Medical Journal 2012; 57: 90-91
}

\section{Introduction}

The recent recognition of anti-N-methyl-D-aspartate receptor (NMDAR) antibody encephalitis has added to the growing list of treatment-responsive, reversible, autoimmune CNS disorders [1]. NMDAR-antibody encephalitis is characterised by a multistage progression from psychosis, memory deficits, seizures and language dysfunction to a state of coma often associated with movement disorders, dysautonomia and breathing instability [2]. Although first described as a paraneoplastic syndrome associated with ovarian teratomata in young women, NMDAR-antibody encephalitis is increasingly recognised in children, often unassociated with tumours [3-5]. This is the first report of NMDAR-antibody encephalitis from Sri Lanka.

\section{Case report}

A 30-month old, previously well girl, presented with two transient focal seizures, which occurred independent of fever. Two days later, paucity of normal movements in the left upper and lower limbs was noted, with intermittent choreiform movements in the same limbs. This was associated with a marked change in behaviour with extreme irritability, self-injurious behaviour, frequent biting of caregivers and severe insomnia. The choreiform movements progressed to hemibalismic movements that resulted in loss of ability to sit even with support. Orofacial dyskinesia, drooling of saliva and inability to swallow, necessitated nasogastric feeding. Over the next two weeks, her speech declined to complete mutism whilst cognition declined from not recognizing her parents to a state of stupor.

Her blood counts, inflammatory markers and sepsis screens were normal. CSF analysis was normal with no evidence of viral antigens or tuberculosis. The electroencephalogram showed initial slowing followed by some sharp wave activity over the right temporal region. MRI of the brain was normal. CT scan of the abdomen excluded ovarian or other tumours. NMDAR antibodies were positive in serum and CSF whilst anti-GAD and antiVGKC antibodies were negative.
She was treated with combined IVIG $(0.4 \mathrm{mg} / \mathrm{kg})$ and intravenous methylprednisolone ( $30 \mathrm{mg} / \mathrm{kg}$ ) for 5 days. Five days after-treatment, she regained eye contact and was able to recognise her parents. She started making simple sounds initially and later single words. The involuntary movements gradually reduced over the next 5-6 weeks with improvement of the hemibalismic movements first and the oro-facial dyskinesia last. A week later, she was able to sit unaided and around 8 weeks post-treatment she was able to ambulate independently. By this time her speech had returned to the pre-illness state. She made a complete recovery after treatment.

\section{Discussion}

Since its original description 3 years ago, NMDARantibody encephalitis has been increasingly recognised worldwide. Children comprise $40 \%$ of all cases [4]. Although uncommon at 30 months of age, the typical features of the syndrome, that include seizures, behavioural abnormalities, involuntary movements, language disintegration and progression to a state of unresponsiveness, suggested the diagnosis of NMDARantibody encephalitis in our patient, which was confirmed by the detection of NMDAR antibodies in serum and CSF. Negative screening tests for sepsis and vasculitis, and the normal MRI of the brain helped to exclude alternative diagnoses.

In adults, NMDAR-antibody encephalitis is characteristically a multistage illness [5]. However, in children, the classical stages of the illness are less distinctive. The prodromal stage in children is likely to present as behavioural changes such as temper-tantrums, hyperactivity and self-injurious behaviour, rather than frank psychosis [4]. In children, recurrent seizures, including status epilepticus are more common than in adults. Prominent autonomic manifestations are less common in children [4].

NMDAR-antibody encephalitis is associated with ovarian teratomata in up to $60 \%$ of women aged 18 years or more [3], but often unassociated with tumours in children [4]. CT scan of abdomen did not detect any tumours in our patient. 
NMDAR antibodies have been shown to substantially reduce the numbers of cell-surface NMDAR in hippocampal neurons in-vitro and in-vivo [2]. Antibodies are thought to internalise the NMDAR by cross-linking the extracellular N-terminal domain of the NR1 subunit [2]. Furthermore, clinical severity of the disease has been shown to parallel the antibody titre in individual patients supporting the likely direct pathogenicity of the NMDAR antibodies [2, 3].

Whilst up to $75 \%$ of patients with NMDAR-antibody encephalitis, with or without associated tumours, showed substantial improvement with early immunotherapy [2], late treatment conferred no benefit [5]. Corticosteroids, intravenous immunoglobulins and plasma exchange are considered first line, whilst rituximab and cyclophosphamide are considered second line immunotherapy. Clinical recovery is usually a multistage process that occurs in the reverse order of symptom development as was demonstrated during the recovery of our patient.

This case report illustrates the good outcome of early immunotherapy and, hence, the need for a high index of clinical suspicion, particularly in children in whom the clinical stages of the illness are less distinctive.

\section{References}

1. Vincent A, Bein C, Irani S, Waters P. Autoantibodies associated with diseases of the CNS: new developments and future challenges. Lancet Neurology 2011; 10: 759-72.

2. Dalmau J, Lancaster E, Martinez-Hernandez E, Rosenfeld MR, Balice-Gordon R. Clinical experience and laboratory investigations in patients with anti-NMDAR encephalitis. Lancet Neurology 2011; 10: 63-74.

3. Dalmau J, Gleichman AJ, Hughes EG, et al. Anti-NMDAreceptor encephalitis: case series and analysis of the effects of antibodies. Lancet Neurology 2008; 7: 1091-8.

4. Florance NR, Davis RL, Lam C, et al. Anti-N-methyl-Daspartate receptor (NMDAR) encephalitis in children and adolescents. Annals of Neurology 2009; 66: 11-8.

5. Irani SR, Bera K, Waters P, et al. N-methyl-D-aspartate antibody encephalitis: temporal progression of clinical and paraclinical observations in a predominantly nonparaneoplastic disorder of both sexes. Brain 2010; 133: 1655-67. 